\title{
Giant Cell Tumor of the Wrist: Rare Location of the Distal Ulna
}

\author{
Siniki Fandebnet ${ }^{1,2}$, Ngamai Kotyade1, A. D. Mahamat Nour ${ }^{2}$, Ngarieguem 0.2, \\ Wassim Brahim Massar²
}

${ }^{1}$ Orthopedics Department, Renaissance University Hospital, N'Djamena, Chad

${ }^{2}$ Faculty of Human Health Sciences, University of N'Djamena, N’Djamena, Chad

Email: *siniki_fandebnet@yahoo.fr

How to cite this paper: Fandebnet, S., Kotyade, N., Nour, A.D.M., Ngarieguem, O. and Massar, W.B. (2021) Giant Cell Tumor of the Wrist: Rare Location of the Distal Ulna. Open Journal of Orthopedics, $11,67-72$.

https://doi.org/10.4236/ojo.2021.113007

Received: January 9, 2021

Accepted: March 9, 2021

Published: March 12, 2021

Copyright $\odot 2021$ by author(s) and Scientific Research Publishing Inc. This work is licensed under the Creative Commons Attribution International License (CC BY 4.0).

http://creativecommons.org/licenses/by/4.0/

\begin{abstract}
Giant cell tumor of the wrist is a rare, benign and usually symptomatic condition. The discovery is sometimes made following a medical imaging examination or a painful symptomatology or more often a visible or palpable swelling with or without vascular and/or nerve compression. At an advanced stage, the $\mathrm{X}$-ray is of paramount importance. The well codified complete surgical resection is part of the therapeutic arsenal. We present a clinical case report of a young woman with a giant cell tumor localized in the wrist in N'Djamena, Chad. This case concerns a 25-year-old patient who presented in July 2020 of a painful swelling lateral to her left wrist bone and whose X-ray radiography showed lysis of the cortical bone in the lower third of the ulna. After the operative resection of the tumor mass, the pathological examination of the operative specimen revealed the diagnosis of a giant cell tumor. A giant cell tumor is a benign condition, with a few symptoms and the location at the ulna is exceptional. Complete surgical resection is a viable treatment option.
\end{abstract}

\section{Keywords}

Giant Cell Tumor, Complete Surgical Resection, Ulna

\section{Introduction}

Giant cell tumors, described since 1811, are benign tumors with a local aggressiveness that are preferentially located in the metaphyseal-epiphysis region of long bones, and a frequent invasion of the subchondral bone [1] [2] [3]. Clinically, the tumor may be asymptomatic, however, it may manifest itself as a swelling, pain, or pathological fracture [2]. Diagnosis is guided by medical imaging and confirmed by histological findings of the bone samples [2]. If left untreated, 
there may be an extension of the osteolysis, destruction of the adjacent joint and pulmonary metastases [2]. Treatment is surgical [2].

We report a case of a 25-year-old woman who presented in July 2020, with a painful swelling lateral to her left wrist bone with an X-ray radiography showing lysis of the cortical bone in the lower third of the ulna. After the operative excision of the tumor mass, the pathological examination of the specimen revealed the diagnosis of a giant cell tumor. With a lytic lesion of the wrist bones in a young woman on $\mathrm{X}$-ray, one must think of a giant cell tumor.

\section{Case Report}

We report the case of a 25-year-old young woman with no notable pathological antecedents who presented at the orthopedic consultation for a painful swelling of the left wrist (Figure 1) that had been evolving for 18 months and without any alteration of the general state. There was no change in color or consistency of the skin with respect to the tumefaction. The biological balance is often normal, however a phosphocalcic balance has been requested to rule out hyperthyroidism.

On physical examination, there was a prono-supination blockage because of the large volume occupied by the tumor mass and the articular destruction at the level of the distal radio-ulnar joint; palmar flexion estimated at $45^{\circ}$ and dorsal extension at $-30^{\circ}$; radial abduction or inclination at $10^{\circ}$ and ulnar adduction or inclination at $25^{\circ}$.

The $\mathrm{x}$-ray showed a lesion with blurred boundaries, extending into the soft tissue that is not limited by a bony shell; with destruction of the cortex, invasion of soft parts and honeycomb pseudo-partitions. And finally the X-ray radiography of the left wrist showed (Figure 2) a lysis of the cortex of the lower extremity of the ulna. It corresponds to grade 3 of the Campanacci and Merle d'Aubignée classification.

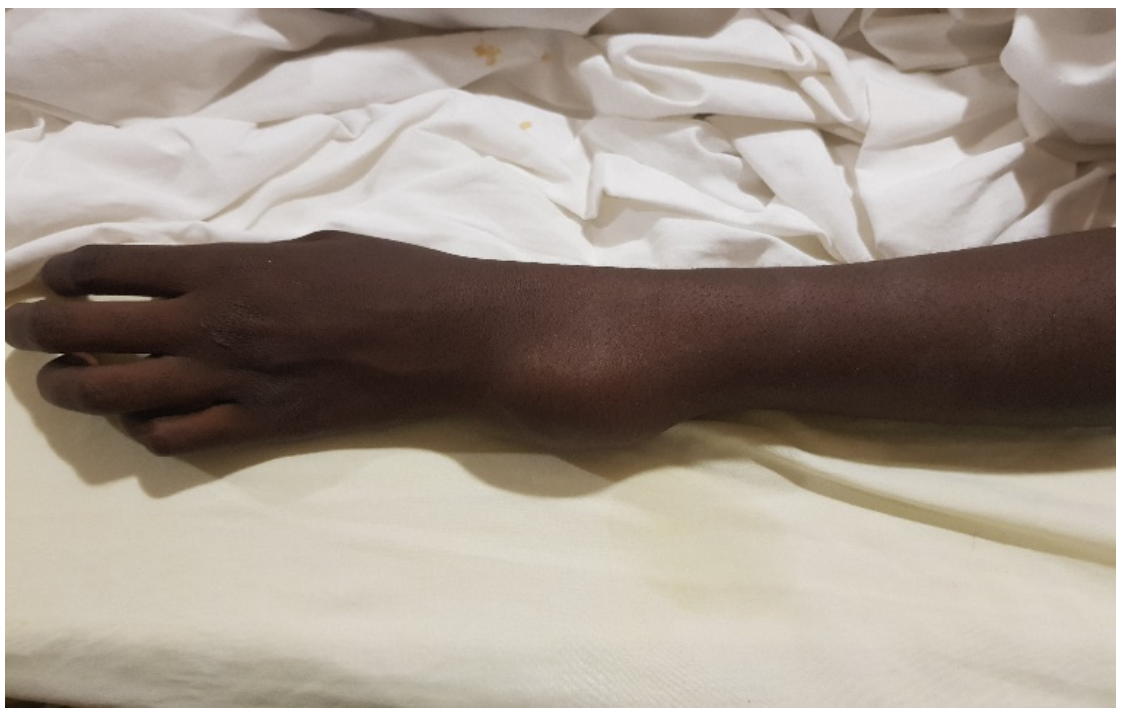

Figure 1. Swelling of the upper outer surface of the left wrist. 


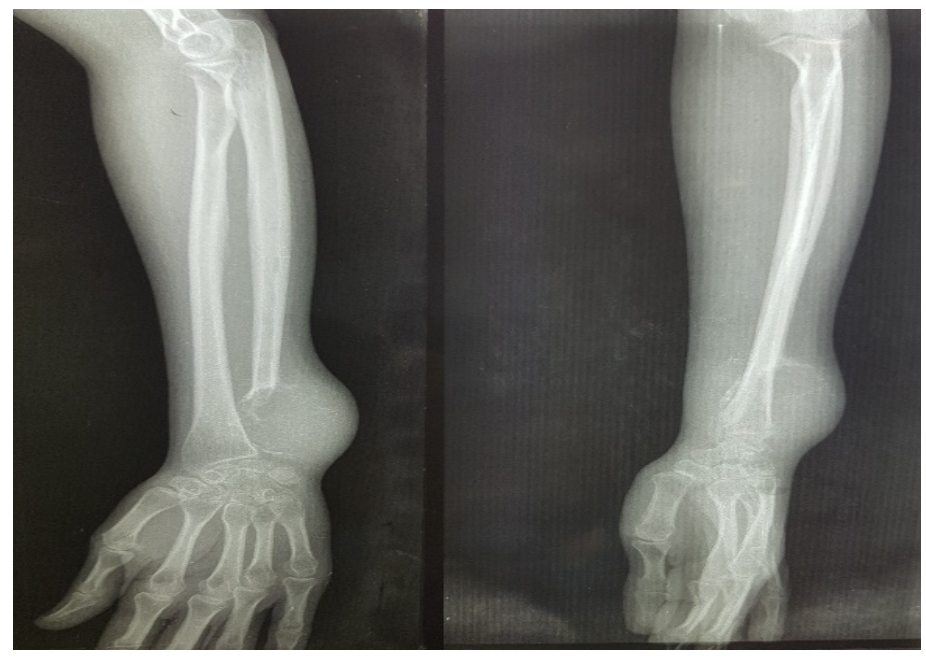

Figure 2. X-ray of the left wrist from the front and in profile showing a lytic lesion.

A complete surgical resection was offered to the patient. Under locoregional anesthesia, an incision on the ulnar border centered on the tumor mass was made. This incision was widened proximally to expose a healthy portion of the cubital bone. A careful dissection of the tumor mass carrying the shell was performed (Figure 3). Surgical removal of the tumor mass $(5 \mathrm{~cm} \times 3 \mathrm{~cm})$ in all its limits, followed by a distal resection of the ulna bone by about $2 \mathrm{~cm}$ in the healthy zone (Figure 3), while leaving the ulnar cartilage distally. A temporary transverse radio-ulnar pin of about $5 \mathrm{~cm}$ was inserted, fixing the free part of the ulna to the radius (Figure 4). Reinforcement with a cast splint was made to complete the distal radio-ulnar stability.

The anatomopathological assessment showed abundant mononuclear cells and discrete nuclear anomalies with marked mitotic activity, but without atypical forms. The histological examination of the bone fragments confirmed a grade 2 giant cell tumor according to Sanerkin, Jaffe Lichtenstein and Pottis.

At three weeks the pin was removed, physical examination showed recovery of the prono-supination (Figure 5) of the palmar flexion and dorsal extension. Surgical treatment with the excision of the large tumor mass improved the function of the wrist and forearm.

\section{Discussion}

Giant cell tumors or formerly called myeloplax tumors are osteolytic lesions; usually located in the epiphyseal areas of long bones. Giant cell tumors of the bone are not common. According to Galvan et al., only 240 cases have been described to date, none of which have occurred in literature. In Africa, a few cases have been reported in Morocco [4] [5] [6] [7]. Young aged (peak between 20 to 30 years, after cartilage fusion) females were in line with the literature [2] [7] [8]. However, the initial presentation of a painful localized swelling is common [2] [8] [9]. The site of the ulna has been described but is not the most common location [1] [2] [8] [9], the preferred locations being the distal end of the femur and 


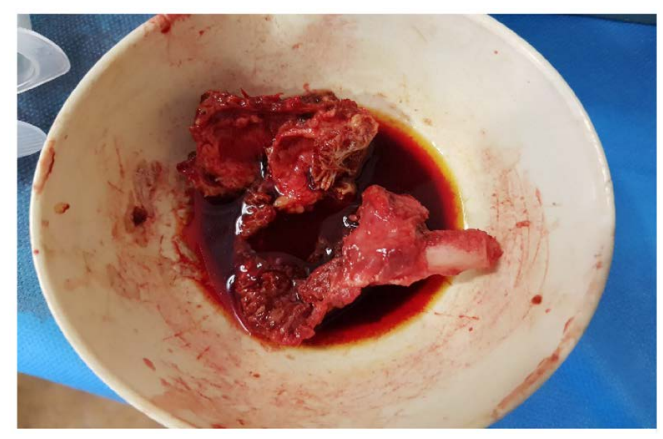

Figure 3. Tumor mass in all its limits with a portion of the ulna bone.

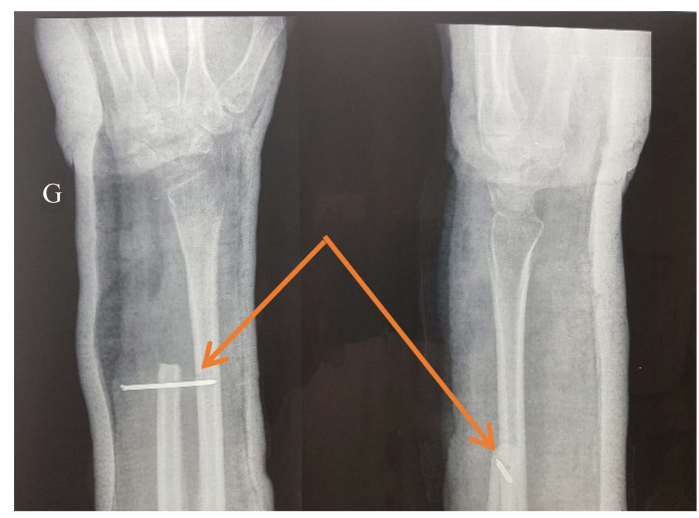

Figure 4. X-ray of the left wrist postoperatively showing the presence of the pin.

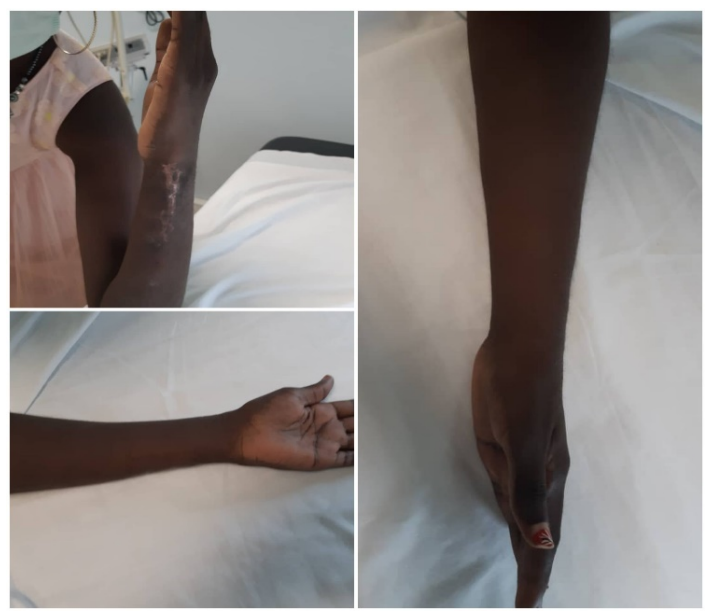

Figure 5. Recovery of pronosupination at 3 months postoperatively.

the proximal end of the tibia [2]. Standard X-ray radiography is performed as a first line with orthogonal incidence [7] [8]. Radiologically, this is a pure lytic lesion at the metaphyseal-epiphysis level [7], which can extend into the subchondral bone [7]. Cortical destruction is associated with pathological fractures during initial presentations [7]. It provides a basis for etiological orientation. It has the advantage of an exhaustive and panoramic view [1] [10]. We can use a CT scan or an MRI study if we fear an invasion of soft parts. In the event of recurrence, a scintigraphy is useful for early diagnosis. Arteriographic embolization is 
necessary in difficult locations and can be a therapeutic procedure (spine, sacrum) [9].

In the past, these tumors were treated by amputation or by major resections and subsequent reconstruction [5].

Currently, surgical treatments are intralesional curettage, curettage and bone grafting, cryotherapy of the cavity after curettage, application of phenol after curettage, radiation, insertion of methyl methacrylate cement into the cavity after curettage, resection followed by an allograft, complete resection with or without reconstruction or stabilization of the ulna and prosthetic reconstruction, and the embolization of the supply vessels [5].

Histological confirmation of the bone samples ensures the diagnosis [2]. When the lesion is localized as in our case, treatment usually consists of surgical tumor excision with a healthy safety margin. This technique has the advantage of preserving joint function, and recurrences are no more frequent than with other techniques according to several authors [2] [7] [9]. After curettage, reconstruction may be done with bone grafts and cementation [7]. Recurrence occurs in up to $20 \%$ of cases [7]. Excision with tumor free margins is associated with lesser recurrence rates. However, for periarticular lesions this is usually accompanied with a suboptimal functional outcome [3]. Various studies suggest that wide resection is associated with a decreased risk of local recurrence when compared with intralesional curettage and may increase the recurrence free survival rate from $84 \%$ to $100 \%$ [1] [2]. However, wide resection is associated with higher rates of surgical complications which led to functional impairment, generally necessitating reconstruction [2]. In other series, the bone resection in the forearm has been filled with the use of autologous vascularized fibula. But this technique is grafted with complications [10]. In our patient's case, we performed an intralesional curettage followed by bone resection of the partially healthy ulna by $2 \mathrm{~cm}$ to minimize the risk of recurrence, followed by the insertion of a temporary transverse radio-ulnar stabilization pin. This procedure resulted in good function preserving wrist joint function after removal of the pin 3 weeks later. After a 5 months follow up, there was no recurrence or functional sequelae of the wrist. Giant cell bone tumors generally have a good prognosis [7].

\section{Conclusion}

The giant cell tumor of the ulna bone, although rare, does not present any particularity. The gold standard X-ray image guided radiography with the bone tissue histology confirmed the diagnosis. Surgical treatment preserved joint function. This should be considered when presented with a lytic epiphyseal bone lesion in a young woman.

\section{Informed Consent}

An informed written consent was obtained from the patient for publication of this report and any accompanying images. 


\section{Conflicts of Interest}

The authors declare no conflicts of interest regarding the publication of this paper.

\section{Contributions from Authors}

All authors contributed to the conduct of this work. All authors also declared that they have read and approved the final version of this manuscript.

\section{References}

[1] Stan, G., Orban, H. and Gheorghiu, N. (2016) Giant Cell Tumor of Long Bones Outcomes of Treatment Corelating with Histopathological Grade. Maedica (Bucur), 11, 296-298.

[2] Sobti, A., Agrawal, P., Agarwala, S. and Agarwal, M. (2016) Giant Cell Tumor of Bone-An Overview. The Archives of Bone and Joint Surgery, 4, 2-9.

[3] Barik, S., Jain, A., Ahmad, S. and Singh, V. (2020) Functional Outcome in Giant Cell Tumor of Distal Radius Treated with Excision and Fibular Arthroplasty: A Case Series. European Journal of Orthopaedic Surgery \& Traumatology, 30, 1109-1117. https://doi.org/10.1007/s00590-020-02679-2

[4] Hammas, N., Laila, C., Youssef, A.L.M., Hind, E.F., Harmouch, T., Siham, T. and Afaf, A. (2012) Can p63 Serve as a Biomarker for Giant Cell Tumor of Bone? A Moroccan Experience. Diagnostic Pathology, 7, Article No. 130. https://doi.org/10.1186/1746-1596-7-130

[5] Ayoub, M.S., Abdel-Salam, F.M. and Abdel-Azim, A.M. (1995) Clinical, Statistical and Immunocytochemical Characterization of Gppuiant Cell Tumour of Bone among Egyptian Population. Egyptian Dental Journal, 41, 1203-1209.

[6] Bai, W.Z.B., Guo, S.B., Zhao, W., Yu, X.C., Xu, M., Zheng, K., Hu, Y.C., Wang, F. and Zhang, G.C. (2019) Comparison of Outcomes of 2 Surgical Treatments for Proximal Humerus Giant Cell Tumors: A Multicenter Retrospective Study. Journal of Shoulder and Elbow Surgery, 28, 2103-2112. https://doi.org/10.1016/j.jse.2019.04.010

[7] Galvan, D., Mullins, C., Dudrey, E., Kafchinski, L., Laks, S. (2020) Giant Cell Tumor of the Talus: A Case Report. Radiology Case Reports, 15, 825-831. https://doi.org/10.1016/j.radcr.2020.03.016

[8] Andreas, F.M., Vasileios, G.I., Panayiotis, D.M., Georgios, N.P., Panayiotis, J.P. and Panayotis, N.S. (2017) Giant Cell Tumor of Bone Revisited. SICOT-J, 3, 54. https://doi.org/10.1051/sicotj/2017041

[9] Youssef, N., Issam, S., Idrissi, K.K., Hicham, S. and Farid, G. (2015) Localisation humérale d'une tumeur à cellules géantes récidivantes (à propos d'un cas). Pan African Medical Journal, 20, 12. https://doi.org/10.11604/pamj.2015.20.12.5519

[10] Raghav, S., Kamal, B., Vikas, B., Aditya, K.M., Mandeep, S.D. and Shivinder, S.G. (2011). En Bloc Excision and Autogenous Fibular Reconstruction for Aggressive Giant Cell Tumor of Distal Radius: A Report of 12 Cases and Review of Literature. Journal of Orthopaedic Surgery and Research, 8, Article No. 14. https://doi.org/10.1186/1749-799X-6-14 